\title{
Real-Time Health Monitoring System with Hybrid Wearable Suit
}

\author{
Anil P. Nautiyal ${ }^{1}$, Manoj Baghari ${ }^{2}$ \\ ${ }^{1,2}$ BRP Science, SAS Uttarkashi, Uttarakhand, India, \\ ${ }^{1}$ anilnautiyal_76@rediffmail.com, ${ }^{2}$ manojbaghari123@gmail.com
}

\begin{abstract}
This paper is aimed to use new technology for monitoring the real time physiological parameter. Sensor network, while born on other application, are the main component of our wearable suit, which is the key step for the smart healthcare. In our research, our main focus is on real time and continuous monitoring of particular person. In biomedical science data monitoring of a subject (person) is very important for which experimental results suggest a physical powerful potential for sensor network due to the low cost and adhoc use of these sensors. The wearable suit will not only to improve the health monitoring but also it will help in disaster sites to find out the victims. We predict that our wearable suit will help greatly to improve the quality of rescue operation, health monitoring and life of a person.
\end{abstract}

Keywords: Physiological Parameter, Electrode Network, Hybrid Device

\section{Introduction}

Hybrid physiological sensor network permit data gathering with the help of surface electrodes, which can be computed very easily [1]. In current era of technology the sensor technologies have got so advanced that we are in almost every field like science, engineering, transport, medicine, monitoring of dependent people, and many other areas. Wearable suit will certainly be helpful to monitor the real time health parameters. There is a hybrid physical network for monitoring all the parameter like EEG (Electroencephalograph), EMG (electromyography), ECG (Electrocardiogram), EOG (Electro Oculogram) [2][3][4][5][6]. Information technology (IT) and artificial intelligence, together can come with a new solution for biomedical engineering. Increasing elderly population in developed countries is one of the biggest challenges. According to the population reference bureau in the next 20 years, the maximum population share will be of over age 65 group, which indicates the need of quality and smart health care to avoid the one the biggest problem we can face in nearby future. Integration of sensor network and consumer electronics can be the one hopeful application in this area. With the help of our wearable suit we can information for the patient. The system will provide services to the elderly populated society as well as the society in which both the parents have to go for the work and cannot give time to the their babies and children. To get the better and smart healthcare researchers in different fields to make the smart healthcare possible. For the wireless transmission one can be use GSM, RFID, Bluetooth, ZigBee, and wireless sensor networks. Development of small or wearable sensor devices will provide with much smart and more mature healthcare services[7][8]. Lots of efforts have been done in development of small sensor devices, which can be integrated in any other substances to implanted in user body.

Article history:

Received (February 3, 2019), Review Result (April 27, 2019), Accepted (August 9, 2019) 
Researcher may find a wonderful survey on wireless sensor networks for health care. In the disaster, lots of people lose their relatives, whose bodies are hard to find. In june, 2013 a massive disaster took place in Uttarakhand, India. In which 5,748 people got there death and almost 4,200 villages got effected. Our system will help to find out the lost bodies in these area which will improve the quality of rescue operations.

In this paper we provide a smart way of healthcare, which can will certainly be beneficial in disaster areas and old age homes.

\section{Description of the work}

All In this paper, we developed a hybrid physiological wearable network system for smart monitoring of healthcare while stabilizing ones comfort and privacy, the wearable suit continuously monitor one's health. Our hybrid physiological network system will work as follows

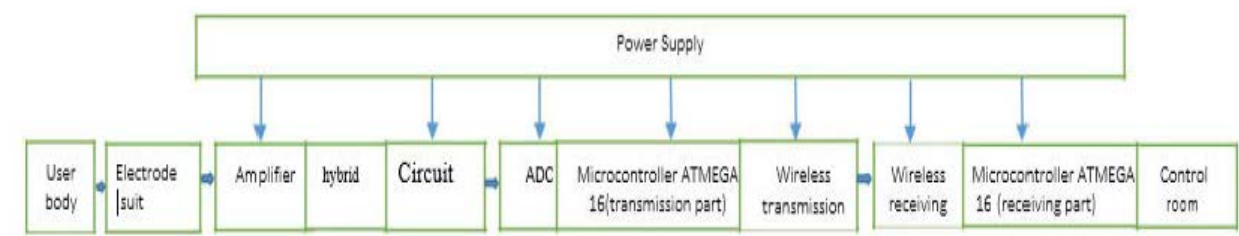

Figure 1. Block diagram of the hybrid network

The block diagram shows how our hybrid network will work. This block diagram consist of the whole system which is consist of the master and slave unit.

\subsection{Master unit}

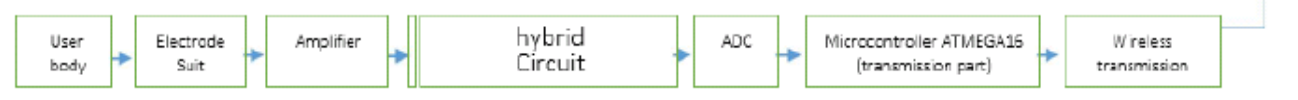

Figure 2. Block diagram of master unit

Master unit is the primary part of our project, which consist of surface electrode connect to the user body. It also contain the pre amplification circuit followed by the hybrid circuit which goes to the ADC before reaching at the microcontroller ATMEGA 16. This data is transmitted through wireless network system to the control room.

\subsection{Slave unit}

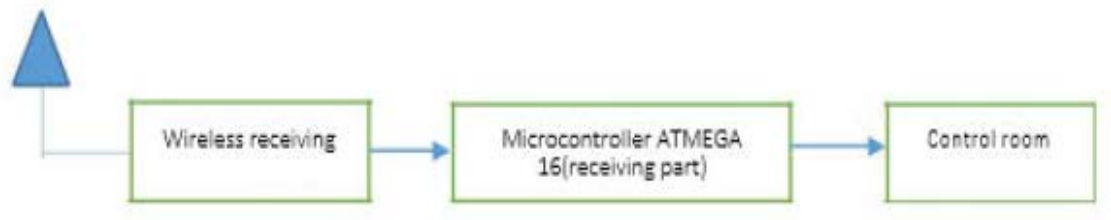

Figure 3. Block diagram of the slave unit 
Slave unit works when it receive the transmitted signal from the master unit. It is basically consist of the wireless receiver module followed by the microcontroller. Then the data is shown at pc as per of the setup of control room, which is shown in Figure 3.

Our hybrid physiological network work in the following manner

1. Wearable suit is wear by the user.

2. By the help of the surface electrodes information (signal) comes in the system.

3. The signals get amplified by the pre amplifier used in the network.

4. The amplified signal then comes in the hybrid circuit to get the final results.

5. These results goes in the microcontroller after it passes through the ADC circuit.

6. Microcontroller passes the information to the wireless transmitter.

7. Transmitted signals are received by the wireless receiver and it can be displayed as per of the set up

Flow chart Diagram

Flow diagram of the system is being drawn as per the steps of the work, which is shown in Figure 4. The flow diagram is dipicting the suit of the system and steps in sequence of events till the final service.

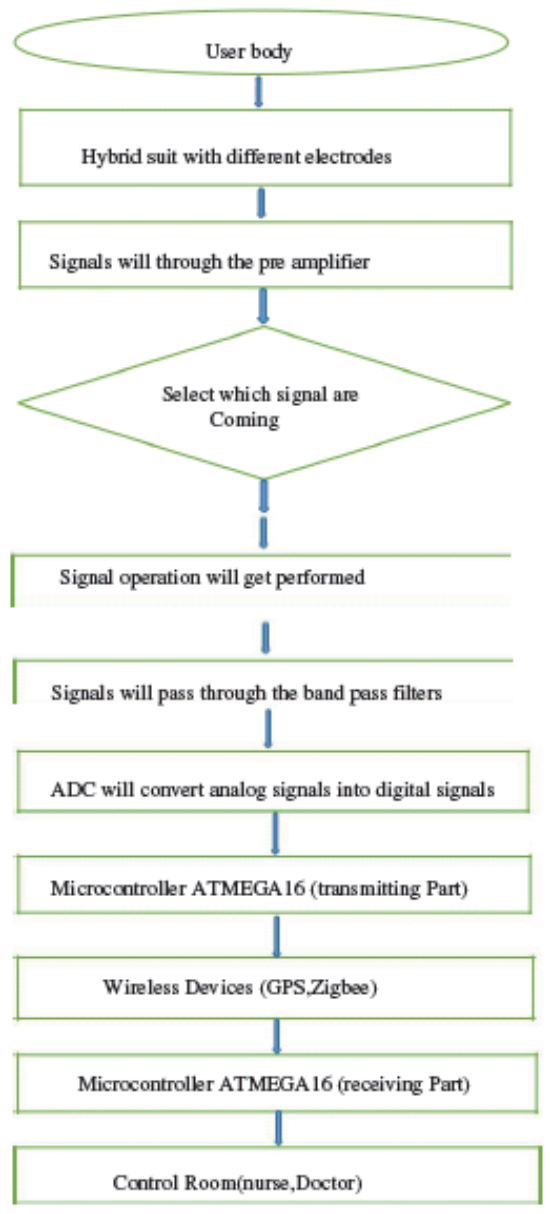

Figure 4. flow chart diagram 


\section{Suit and its Components}

a) Body network

The hybrid physiological network consist of some small portable devices such as heart rate, temperature, oximeter and other sensor as per of the requirement. By the help of this system desired tasks like biophysical monitoring and location detection is done.The devices is very small that can be worm comfortably [9]. The power use is very small that why battery can use for long time.

b) Placing of Electrode

Electrode network is placed on human body and so on to support sensing and monitoring, including heart rate mental processing eye movement mussel's movement. The system work wired and with battery power. Electrodes are placed as per of the requirement of the monitoring.

c) Hardware

The following hardware devices are used in our hybrid physiological network system.

1. surface electrodes

2. Amplifier

3. Switches

4. Analog to digital convertor

5. Microcontroller

6. wireless module(Zigbee,GPS)

\section{Features of Hybrid Physiological Suit}

-Mobility

The hybrid suit collect the data and words at the minimum input from the user for which small devices are used to collect data and communicate. The suit is designed in such a way that it can carried on the body. Since surface electrodes are used for the monitoring, the user can move and work. It is safer and convenient.

-Real-time

A user can be monitored continually, both as Physiological and environmental which allow realtime response to the user about the emergency health status. The data gathered by continuous monitoring have more advantages over the traditional patient monitoring.

-Reconfiguration and self-organization

Since the system is fixed in the wearable suit it is easy to add or remove the sensors directly from the system. It gives the great advantage of Reconfiguration and self-organization.

Data acquisition

a. Body network

We have implemented wearable electrodes in a hybrid suit, the function of this suit is the recording of human activities using the surface electrode. The recorded activity data can be used to compare the past human activates which can be used to monitor the health status.

b. ECG, EMG, EOG, EEG

These electrodes are wearable by which, Heart rate (HR), electrocardiogram (ECG) electro

Encephalography (EEG) electrooculography (EOG) electromyography (EMG) is available and has been shown in Figure 5.

EMG (electromyography) Electromyography is us to check the electrical activities produce by the skeletal muscles which comes under electro diagnostic medicine techniques. The machine which is use to perform Electromyography is known as electromyography, the recording is called electromyogram. EMG signal is weak and with the unwanted noises, before using these signals they undergoes the pre amplifier and post amplifier circuit. 


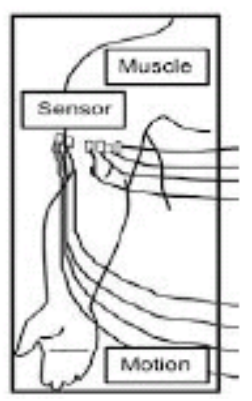

\section{Figure 5. Wearable electrodes}

\section{ECG (Electrocardiogram)}

The machine which is used to measure the heart rate is known as ECG, the heart rate is converted into signal which is taken on a paper or on a monitor. An ECG is a recording by the help of surface electrode generated by the heart. Electrodes are placed at labelled location on the person's body to collect the information for the ECG measurement. ECG is used to measure and diagnose irregular rhythms of the heart, particularly irregular rhythms which can be caused by damage to the conductive tissue which carries electrical signals, or irregular rhythms caused by electrolyte imbalances. Damaged heart muscle can be identify wit the help of ECG.

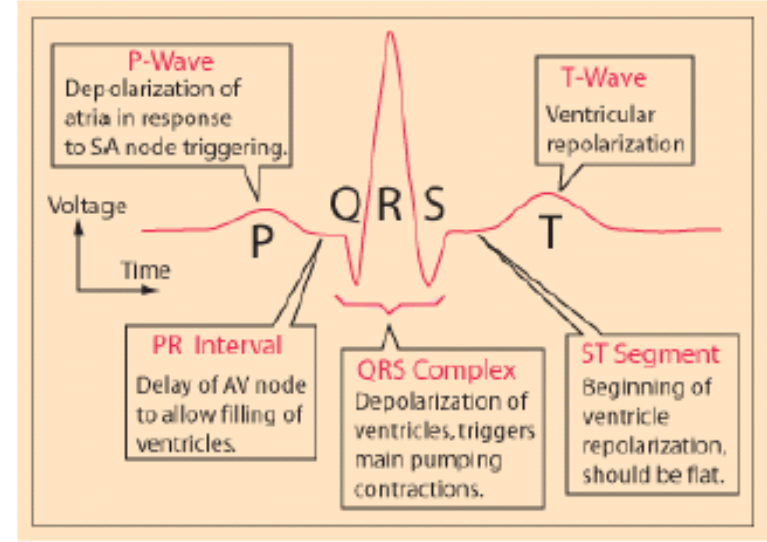

\section{Figure 6. Data Collection using various units}

\section{EEG(Electroencephalograph)}

Small brain wave sensor can be created highlighting the performance Devices known as electroencephalographs, or EEGs, have been used to record brain signals so that it can be reasonable to complete this in the required time frame. Brain singles are classified as $\operatorname{Delta}(0.1 \mathrm{~Hz}-3 \mathrm{~Hz})-$ In this mental state a person stay in deep and dreamless sleep, which is a non-REM sleep state or unconscious state.

Theta $(4 \mathrm{~Hz}-7 \mathrm{~Hz})$ - When a person imagine this single comes into existence. In this state a person's creativity is increased.

Alpha $(8 \mathrm{~Hz}-12 \mathrm{~Hz})$ - when a person eyes get closed, this mental comes into existence, these signals are also known as relaxed signals.

Low Beta (12Hz-15Hz-) In this state a person remain relaxed yet focused.

Midrange Beta(16Hz-20Hz)- In this state on remain in aware of self and surrounding. High Beta $(21 \mathrm{~Hz}-30 \mathrm{~Hz})$ - In this state on remain in alertness state EOG (Electro Oculogram) 
To measure the electrical response of the light-sensitive cells (rods and cones) and motor nerve components of the eye, Electrooculography (EOG) text is done. This involves placing of electrodes on the skin near the eye[9] [10]. The voltage difference between two electrodes is consider as the output of the eye movement, which has been shown in Figure 7.

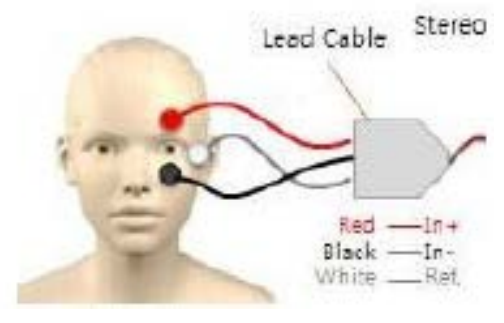

Figure 7. EOG

Wireless system: The strong point of our hybrid network system is a single wireless system which is used as a gateway between the wearable suit and nurse station. Suit can use a Zigbee, GPS and other wireless protocol for communication.

Data mining and management: Input is taken by the help of surface electrodes, which is then transmitted by the help of wireless module to the PC connected in the control room, where all the information can be stored coming from hybrid suit.

Graphical user interfaces (GUI): Monitoring body, services provider, and technicians need different requirements. The system offers two different GUIs, one which is located on the user body, by which information from the user body is gathered. A second GUI, which is located in the nurse room and its gets all the monitoring facilities.

\section{Discussion and Outcome}

Data association permits to identify the right person among a group when a person is responsible for a triggered event. By the help of data association one can know, who is doing what?, in a society without biometric identification. The elderly population is increasing very rapidly in developed countries, this is why it is very useful to avoid the errors in medical science in near future.

Economic benefit

We are using a single circuit to calculate the EEG, EMG, ECG, EOG which is the greatest move with respect to the other approaches we are using in present era. Using our concept of suit economical as well as the technical up gradation are possible. Instant of using different machine, a single circuit gives us the benefit to reduce the bulkiness. It is easy to carry that system to the different location.

Cost of the individual machines

EEG MACHINE MAXIMUS 32 CHANNEL COST IS RS 210000.ECG Machine - Single Channel cost is RS20000. EMG with NCV cost is RS 1.5lack. EOG machine cost is RS 15000.

Individually these machine are getting much cost relative to the electrowear suit we are proposing.

OUR PROJECT IS BENEFICIAL IN DIFFERNRNT AREAS WHICH IS AS

Patient - A general patient cannot be get into surveillance by a nurse or a doctor. But with the concept of our hybrid suit one can get the 24 hour surveillance.

Military - our soldier works in so difficult location like siyachin where survival for common person is not possible. Their health get effected by that location. By the help of our project it will be very easy to monitor their health second to second. 
Coal mining workers - coalmining workers works in that difficult location where they get effected so easily. We can monitor their health very easily.

Mountaineers, Dope diving and other adventurous sports- it will be very easy to get instant health report of the person who is participating in risky situation.

Spiritual visitor - our electrowear suit will give benefit to those who are under the surveillance to doctors yet wants go to the spiritual visits which may be dangerous for their life. With the help of our suit one can get 24 hours monitoring of their health.

\section{Conclusion and Future Scope}

The proposed hybrid suit will be beneficial for patient, military, coal mining workers, mountaineers, dope diving, adventures sports, spiritual visit etc. In near future, our hybrid suit will facilitate to the different user for continuous monitoring of their health. In the way of smart healthcare, our concept will play a key role in development of the smart devices. The concepts, which have been considered in the work are compatible and scalable hence can be used for other such designs too.

In future, to increase the quality of data gathering, sensor's efficacy and data association algorithm are the two important component which must be develop. Our suit is designed in such a manner that it will monitor and collect the person data without disturbing his/her privacy.

\section{References}

[1] Gaurav Kumar Jaiswal, Ranbir Paul, "ECG Classification with the help of Neural Network” International Journal of Electrical and Electronics Research” Vol.2, Issue2, pp 42-46, April-June (2014)

[2] S.K arpagachelvi, Dr.M. Arthanari, M.Sivakumar ECG Feature Extraction Techniques- A Survey Approach”, International Journal of Computer Science and Information Security, Vol. 8, No.1, April (2010)

[3] Rajiv Ranjan, V.K. Giri, “A Unified Approach of ECG Signal Analysis” International Journal of Soft Computing and Engineering ISSN: 2231-2307, Vol.2, Issue.3, July (2012).

[4] Leif Sornmo, Pablo Laguna, "Electrocardiogram (ECG) Signal Processing” Wiley Encyclopedia of Biomedical Engineering, Copyright 2006 John Wiley \& Sons, Inc DOI: 10.1002/9780471740360.ebs1482

[5] R. Chavarriaga and J. d. R. Mill'an, "Learning from EEG error-related potentials in noninvasive brainComputer interfaces,” IEEE Transactions on Neural Systems and Rehabilitation Engineering, Vol.18, No.4, pp. 381-388, Aug (2010).DOI: 10.1109/TNSRE.2010.2053387

[6] R. Ramli, H. Arof, F. Ibrahim, N. Mokhtar, and M. Y. I. Idris, "Using finite state machine and a hybrid of EEG signal and EOG artifacts for an asynchronous wheelchair navigation,” Expert Systems with Applications, vol. 42, no. 5, pp. 2451-2463, (2015). DOI: 10.1016/j.eswa.2014.10.052

[7] Hansen D. W., Hansen, J. P., Nielsen, M., Johansen, A. S. and Stegmann M. B, "Eye Typing using Markov and Active Appearance Models”, IEEE Workshop of Applications on Computer Vision, Orlando, FL, USA, (2002), pp.32-136 DOI: 10.1109/ACV.2002.1182170

[8] Satish Kumar, Adyasha Dash, Manoj Kumar Mukul, "Design and Development of Low-Cost EOG Acquisition Circuit for HMI Application”, 2015 2nd International Conference on Signal Processing and Integrated Networks (SPIN).DOI: 10.1109/SPIN.2015.7095398

[9] Jaakko Malmivuo, Robert Plonsey, "Bioelectromagnetism Principles and Applications of Bioelectric and Biomagnetic Fields”, Oxford University Press,(1995), Chapter 28, pp. 437-441 DOI: 10.1093/acprof:oso/9780195058239.001.0001

[10] A Saravanan et al. , "Design and implementation of Low power, Cost effective Human Machine Interface by Left and Right Eye Movement Technique”,978-1-4673-7910-6/15 @2015 IEEE. 
Real-Time Health Monitoring System with Hybrid Wearable Suit

[11] Yasir Hafeez, Syed Saad Azhar Ali, Aamir Saeed Malik. "Neurofeedback training content for treatment of stress", 2016 IEEE EMBS Conference on Biomedical Engineering and Sciences (IECBES), (2016) DOI: 10.1109/IECBES.2016.7843429 